\title{
Prognostic Significance of Host-related Biomarkers for Survival in Patients with Advanced Non-Small Cell Lung Cancer
}

\author{
Sookyung Lee ${ }^{\bowtie}$, Wankyu Eo², Hyeonjin Jeon³, Sora Park³ ${ }^{3}$ Jean Chae ${ }^{3}$ \\ 1. Depart of Clinical Oncology, College of Korean Medicine, Kyung Hee University; \\ 2. Depart of Medical Oncology and Hematology, College of Medicine, Kyung Hee University; \\ 3. Department of Clinical Korean Medicine, Graduate School, Kyung Hee University.
}

$\triangle$ Corresponding author: Sookyung Lee, Department of Clinical Oncology, College of Korean Medicine, Kyung Hee University Hospital at Gangdong, 892 Dongnam-ro, Gangdong-gu, Seoul, 05278, Republic of Korea Tel:+82-2-440-6229 Fax:+82-2-440-7267 e-mail: sookyung@khu.ac.kr

(c) Ivyspring International Publisher. This is an open access article distributed under the terms of the Creative Commons Attribution (CC BY-NC) license (https://creativecommons.org/licenses/by-nc/4.0/). See http://ivyspring.com/terms for full terms and conditions.

Received: 2017.05.07; Accepted: 2017.07.05; Published: 2017.08.25

\begin{abstract}
Objective: This study identified host-related prognostic biomarkers for survival in patients with advanced non-small cell lung cancer (NSCLC).

Methods: This study was based on the retrospective review of the medical records of 135 patients with pathologically confirmed advanced NSCLC. The host-related biomarkers assessed in this study that reflected patient condition included hemoglobin $(\mathrm{Hb})$ levels; platelet $(\mathrm{PLT})$, neutrophil, lymphocyte, and monocyte counts; and ferritin concentrations. The overall survival (OS) was calculated by Kaplan-Meier analysis and compared using log-rank tests. Univariate and multivariate analyses of Cox proportional hazards regression were used to evaluate the prognostic impact for survival.

Results: Of the enrolled patients, $91.1 \%$ had stage IV NSCLC, $42.2 \%$ had ECOG-PS scores of 2, and 57\% had undergone multiple rounds of prior systemic therapy. The prognostic factors included low $\mathrm{Hb}$ concentration (men: $\mathrm{Hb}<13 \mathrm{~g} / \mathrm{dL}$, women: $\mathrm{Hb}<12 \mathrm{~g} / \mathrm{dL} ; p=0.046$ ), increased neutrophil count (> $7,700$ cells $/ \mu L ; p<0.001)$, decreased lymphocyte count $(\leq 1500$ cells $/ \mu L ; p=0.011)$, increased monocyte count ( $>800$ cells $/ \mu \mathrm{L} ; p<0.001$ ), and high ferritin level (men: $>200 \mathrm{ng} / \mathrm{mL}$, women: $>150$ $\mathrm{ng} / \mathrm{mL} ; p<0.001)$, which were associated with poor OS and increased hazard of mortality. The multivariate proportional hazards model revealed that lymphocyte count, monocyte count, and ferritin level were independent host-related prognostic biomarkers for survival. Increased monocyte count (HR, 3.15; 95\% Cl, 1.64-6.04; $p<0.001)$ and high ferritin level $(\mathrm{HR}, 1.81 ; 95 \% \mathrm{Cl}, 1.24-2.64 ; p=0.002)$ were significantly associated with poor survival, whereas increased lymphocyte count $(\mathrm{HR}, 0.57 ; 95 \%$ $\mathrm{Cl}, 0.40-0.83 ; p=0.004$ ) showed prolonged survival.

Conclusion: Immune factors, such as lymphocyte and monocyte counts, as well as serum ferritin levels, are significant host-related prognostic biomarkers for survival with direct relevance to survival time in patients with advanced NSCLC.
\end{abstract}

Key words: non-small cell lung cancer (NSCLC); prognosis; biomarkers; survival.

\section{Introduction}

Lung cancer is the leading cause of death, representing $19.4 \%$ of all cancer deaths worldwide. ${ }^{1}$ Non-small cell lung cancer (NSCLC) accounts for approximately $85 \%$ of lung cancers, ${ }^{2}$ and most NSCLC patients have unresectable disease at initial diagnosis due to advanced stage or metastasis. ${ }^{3}$
Despite systemic therapy for advanced NSCLC, the median overall survival (OS) with palliative chemotherapy is less than 1 year, ${ }^{4}$ with a 5 -year relative survival rate of distant NSCLC at diagnosis of $4.3 \%$ in the US. ${ }^{5}$

Recently, immunotherapy focusing on patient 
immune checkpoint inhibitors has been recommended for the treatment of advanced NSCLC. ${ }^{6}$ This clinical application of immunotherapy changes cancer treatment to focus on host-related therapy based on the interactions between tumor and host. ${ }^{7,8}$ The importance of these interactions is emphasized by the genomic diversity evident in various genomic alterations, intra-tumor heterogeneity, and genetic instability. ${ }^{9}$ This recent progress in immunotherapy and the recent findings of genomic diversity have highlighted the emerging importance of the patient hosts of the tumors.

Despite the availability of therapeutic agents that reportedly prolong progression-free survival (PFS), indicating their clinical benefits, treatment decisions for the systemic therapy of advanced NSCLC remain a challenge for prolonging overall survival (OS)..$^{10}$ The clinical practice of the treatment of advanced NSCLC is a continuation of the decisions required for optimal care: choosing therapeutic regimens and judging the transition time from palliative to supportive care based on consideration of the treatment benefits and prognostic predictions. The patient is the crucial factor in every decision and judgment. The optimal treatment decision and prognosis prediction should be made from the perspective of host-tumor interaction, considering the spread of cancer throughout the body. ${ }^{11}$

Studies on the prognostic factors of NSCLC have mostly been performed in patients in diverse stages or at the initiation of treatment after diagnosis. ${ }^{12}$ The prognostic factors of NSCLC have been equated with the anatomical extent of the tumor and related to tumor response to the initiation of therapy. Identification of the biological markers that reflect the clinical condition of the patient is important for the determination of the optimal treatment and prognosis prediction. Among peripheral blood tests, levels of C-reactive protein and albumin; leukocytes counts; and inflammation scores have been assessed as markers for the prognosis of patients with NSCLC.

One of the most common biomarkers used to monitor an individual's overall health is complete blood cell count, which measures levels of red blood cells, subtypes of white blood cells, platelets, and hemoglobin. Changes in this biomarker indicate the patients' underlying conditions, and can be used to monitor for hematologic abnormalities caused by cancer or therapy in solid tumors as well as hematologic malignancies. Another host-related biomarker candidate, ferritin, is the major iron-binding protein that acts as a buffer of iron balance; serum ferritin concentrations are used as a diagnostic test for iron deficiency anemia. Until now, few studies have assessed biomarkers from the perspective of host-related parameters to compare their prognostic effect for survival with each parameter of blood cell count and ferritin in patients with advanced NSCLC.

Therefore, this study investigated the potential impact of host-related biomarkers such as hemoglobin, leukocyte, platelet, and ferritin levels for survival in advanced NSCLC. The objective of this study was to evaluate the prognostic impact of these host-related biomarkers on survival, and to identify available host-related biomarkers for predicting survival in patients with advanced NSCLC.

\section{Materials and Methods}

This study was a retrospective review of the medical records of patients with advanced NSCLC managed with Korean Medicine (KM). The Institutional Review Board of Kyung Hee University at Gangdong approved this study (IRB no: KHNMCOH 2016-02001).

\section{Eligibility}

Patients with pathologically confirmed advanced NSCLC and over 18 years of age who were managed with KM from 2006 to 2014 were included in this study. Patients who underwent laboratory tests at the initiation of treatment with Eastern Cooperative Oncology Group performance status (ECOG-PS) scores from 0-2 were enrolled. Those with brain metastasis were also included. Patients who were eligible for confirmation survival in the Survival Registry of the Korean Health Insurance Service were enrolled. The exclusion criteria included liver or renal dysfunction, supplemental oxygen, or failure to undergo laboratory tests at treatment initiation. Patients who were at high risk of oncologic emergencies such as cardiac tamponade and superior vena cava syndrome were not eligible for this study. Patients who were concurrently undergoing conventional therapy were excluded. Finally, patients who had undergone chemotherapy or radiotherapy 3 weeks before the time of the baseline laboratory tests were also excluded in order to avoid the confounding effects of conventional therapy.

\section{Variables}

Clinicopathologic characteristics and host-related biomarkers were investigated. The clinicopathologic characteristics of the enrolled patients that we investigated were the ECOG-PS score, the cell type, the tumor stage, the number of organs with metastasis, and any prior treatment modalities used. For the identification of host-related biomarkers useful for predicting survival, the major biologic markers used to reflect patient condition, 
including hemoglobin $(\mathrm{Hb})$ concentration, platelet (PLT) count, absolute neutrophil count (ANC), absolute lymphocyte count (ALC), absolute monocyte count (AMC), and serum ferritin concentration, were assessed.

White blood cell count, platelet count, and hemoglobin concentration were measured using an automated hematology system (XN-9000, Sysmex, US). Serum ferritin concentration was analyzed using an electrochemiluminescence immunoassay (Modular E170, Roche Diagnostics, Germany).

The continuous variables of prognostic factors were dichotomized based on cut-off values determined according to the reference values for hematologic abnormalities. The $\mathrm{Hb}$ cut-off concentrations were $12 \mathrm{~g} / \mathrm{dL}$ for women and $13 \mathrm{~g} / \mathrm{dL}$ for men, which are the common cut-offs used in anemia studies. ${ }^{13}$ The PLT cut-off concentration was $400 \times 10^{3} / \mu \mathrm{L}$, which is used as the criteria for thrombocytosis. ${ }^{14}$ Regarding the leukocytes, the ANC cut-off concentration was 7,700 cells $/ \mu \mathrm{L}$ based on the criteria for neutrophilia; ${ }^{15}$ similarly, the ALC cut-off was 1,500 cells $/ \mu \mathrm{L}$, based on the criteria for lymphocytopenia, ${ }^{16}$ and AMC cut-off was 800 cells $/ \mu \mathrm{L}$ based on the criteria for monocytosis. ${ }^{17}$ The serum ferritin cut-off value was $200 \mathrm{ng} / \mathrm{mL}$ in men and $150 \mathrm{ng} / \mathrm{mL}$ in women, which indicate a severe risk of iron overload according to the World Health Organization (WHO) serum ferritin concentration guidelines. $^{18}$

\section{Treatment}

Patients were managed with $\mathrm{KM}$, including administration of herbal medicine and acupuncture. The main anticancer agent used in this study was the extract of Rhus verniciflua Stokes (RVS), a lacquer tree that grows in East Asia. RVS has been reported to have antiproliferative, apoptotic, anti-angiogenic, and anti-tumor migration effects on various cancer types. ${ }^{19-23}$ RVS was extracted with water using standard methods to deplete the toxic allergen urushiol, and was then concentrated and lyophilized into a powdered form. The content of the final RVS extract was examined, to ensure the absence of urushiol and to quantify any other compounds present, in order to maintain consistent quality. The reported clinical effects of RVS on advanced NSCLC include prolonged OS when used as a combined therapy with first-line chemotherapy, as a maintenance therapy after first-line chemotherapy, and as a second-line treatment. ${ }^{24,25}$ In this study, RVS extract was administered orally at a dosage of three $500 \mathrm{mg}$ capsules per day.

\section{Overall Survival}

In this study, OS was defined as the time from the initiation of KM treatment to the time of death, which could be brought about by any cause. When patients were lost to follow-up or if death was not recorded, the patient was censored. The survival time of censored patients was defined as the period from treatment initiation to the last day of follow-up or to the date on which survival was investigated.

\section{Statistical Analysis}

The clinicopathologic characteristics of the patients were analyzed by descriptive analysis. Survival time was estimated using the Kaplan-Meier method and compared with log-rank tests. Cox proportional hazard models were used to identify the prognostic factors for survival. Univariate analysis was performed for each potential prognostic factor. After eliminating confounding factors due to sex, age, ECOG-PS, and cell type, multivariate analysis was performed using the statistically significant factors from the univariate analysis (factors with $p$-values less than 0.05). To assess the relationship between prognostic factors, Pearson's correlation coefficients were measured, and subgroup analyses were conducted using chi-squared tests and Wilcoxon signed-rank tests. $P$-values less than 0.05 were considered statistically significant. The statistical analyses were conducted using SAS E-guide 7.1 software (SAS Institute, Cary, NC).

\section{Results}

A total of 135 patients met the eligibility criteria of being pathologically confirmed as having advanced NSCLC, having ECOG scores less than 3, and being treated only with KM (Figure 1). Their clinicopathologic characteristics and host-related biomarkers are summarized in Table 1.

Of the 135 enrolled patients, 78 (57.8\%) were men and $55(40.7 \%)$ were over 65 years of age. The majority of patients $(91.9 \%)$ had stage IV disease and had more than one organ with metastasis. Adenocarcinoma and squamous cell carcinoma accounted for $68.1 \%$ and $21.5 \%$ of cases, respectively. Seventy-seven patients $(57 \%)$ had undergone at least one prior chemotherapy regimen and 58 patients $(43 \%)$ were chemotherapy-naïve. The median duration time to initiate $\mathrm{KM}$ treatment was 4.8 months after initial NSCLC diagnosis and 3.6 months after metastatic NSCLC diagnosis.

The median OS of the enrolled patients was 11.5 months (95\% CI, 10.3-14.8 months). Compared to the survival curve based on the concentration of host-related biomarkers, $\mathrm{Hb}, \mathrm{ANC}, \mathrm{ALC}, \mathrm{AMC}$, and ferritin showed significant differences in OS, whereas 
PLT concentration was not associated with survival. The median OS of patients with anemia was 9.8 months, compared to 13.8 months in patients without anemia $(p=0.045)$. Among leukocyte subtypes, the ANC, ALC, and AMC were associated with survival. The median OS of patients with increased ANC was 3.7 months, while the median OS in patients with low ANC was 13.4 months $(p<0.001)$. The median OS of patients with decreased ALC was 9.2 months, compared to 17.7 months in patients with high ALC ( $p$ $=0.011)$. The median OS of patients with increased AMC was 3.5 months, while the median OS in patients with low AMC was 13.2 months $(p<0.001)$. Finally, the median OS of patients with increased serum ferritin concentration was 8.4 months, compared to 16.7 months in patients with low ferritin concentration $(p<0.001)$ (Figure 2$)$.

Assessed for eligibility

$($ Total lung cancer patients $=564)$

Excluded $(N=429)$

Small cell lung cancer $(N=64)$

Stage at initial visit $(N=44)$

No evidence of disease $(N=14)$

Stage I $(N=6)$

Stage II $(N=4)$

Stage IIIa $(N=14)$

Unknown $(N=6)$

Unconfirmed cell type $(N=14)$

No biopsy performed $(N=9)$

No biopsy record $(N=5)$

Not available for survival analysis $(N=16)$

Foreigner $(N=2)$

Restriction of health insurance $(N=14)$

Not treated with any KM treatment $(N=19)$

Concurrently treated with conventional medicine $(N=177)$

Chemotherapy $(N=164)$

Radiation therapy $(N=11)$

CCRT $(N=2)$

ECOG performance status $>2(N=45)$

Missing laboratory test results $(N=50)$

Analysis $(N=135)$

Biopsy-confirmed advanced NSCLC patients treated with Korean Medicine

ECOG 0-2

Full set of laboratory tests (ferritin and complete blood and differential counts)

Figure 1. Flow diagram of the enrolled patients 
Table 1. Characteristics of the enrolled patients.

\begin{tabular}{|c|c|c|c|}
\hline Variables & & $N$ & $(\%)$ \\
\hline \multicolumn{4}{|l|}{ Clinicopathologic Factors } \\
\hline Age (years) & $<65 / \geq 65$ & $80 / 55$ & $59.3 / 40.7$ \\
\hline Sex & Male/female & $78 / 57$ & $57.8 / 42.2$ \\
\hline Smoking & Non-smoker/ex-smoker/smoker & $68 / 65 / 2$ & $50.4 / 48.1 / 1.5$ \\
\hline ECOG-PS & $0 / 1 / 2$ & $8 / 70 / 57$ & $5.9 / 51.9 / 42.2$ \\
\hline Stage & IIIB/IV & $12 / 123$ & $8.9 / 91.1$ \\
\hline Cell type & Adenocarcinoma/squamous cell carcinoma/others & $92 / 29 / 14$ & $68.1 / 21.5 / 10.4$ \\
\hline Number of organs with metastasis & $0 / 1 / 2 / 3$ & $12 / 54 / 39 / 30$ & $8.9 / 40.0 / 28.9 / 22.2$ \\
\hline Prior surgery & No/yes & $102 / 33$ & $75.6 / 24.4$ \\
\hline Prior chemotherapy & None/first line/second line/ $\geq$ third line & $58 / 38 / 13 / 26$ & $43.0 / 28.1 / 9.6 / 19.3$ \\
\hline Prior radiotherapy & No/yes & $86 / 49$ & $63.7 / 36.3$ \\
\hline \multicolumn{4}{|l|}{ Host-related Biomarkers } \\
\hline \multirow[t]{2}{*}{ Ferritin (ng/mL) } & Men $\leq 200 />200$ & $35 / 43$ & $44.9 / 55.1$ \\
\hline & Women $\leq 150 />150$ & $32 / 25$ & $56.1 / 43.9$ \\
\hline \multirow[t]{2}{*}{ Hemoglobin (g/dL) } & Men $<13 / \geq 13$ & $43 / 35$ & $55.1 / 44.9$ \\
\hline & Women $<12 / \geq 12$ & $35 / 21$ & $62.5 / 37.5$ \\
\hline Platelet $\left(\times 10^{3} / \mu \mathrm{L}\right)$ & $<400 / \geq 400$ & $123 / 12$ & $91.1 / 8.9$ \\
\hline Neutrophil (cells/ $\mu \mathrm{L}$ ) & $\leq 7700 />7700$ & $117 / 18$ & $86.7 / 13.3$ \\
\hline Lymphocyte (cells/ $\mu \mathrm{L})$ & $<1500 / \geq 1500$ & $78 / 57$ & $57.8 / 42.2$ \\
\hline Monocyte (cells $/ \mu \mathrm{L})$ & $\leq 800 />800$ & $122 / 13$ & $90.4 / 9.6$ \\
\hline
\end{tabular}

ECOG-PS = Eastern Cooperative Oncology Group performance status

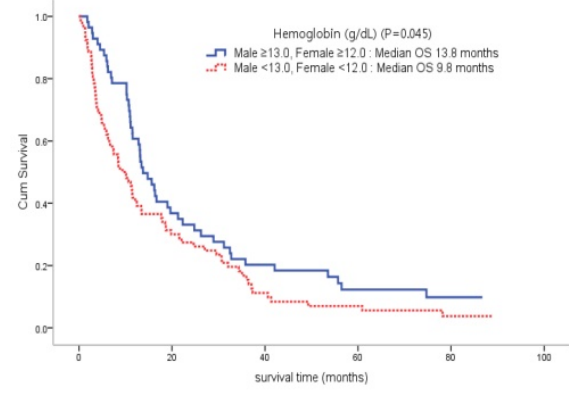

(A)

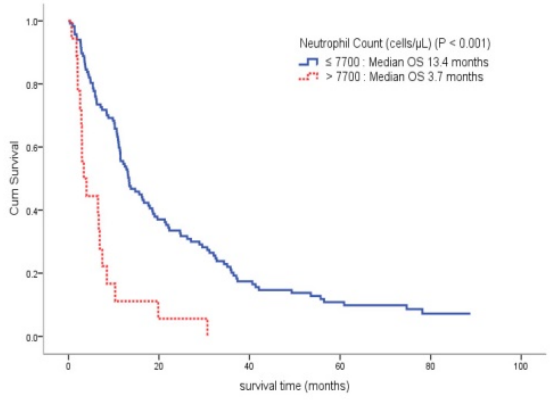

(D)

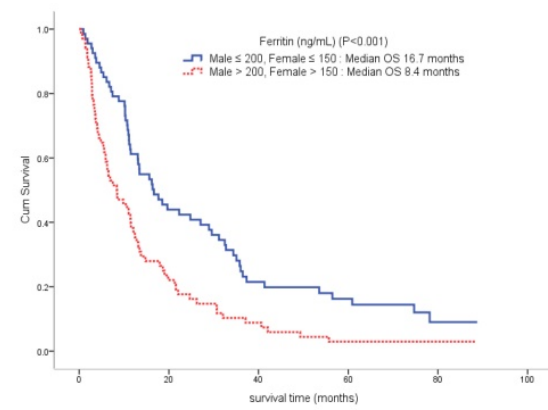

(B)

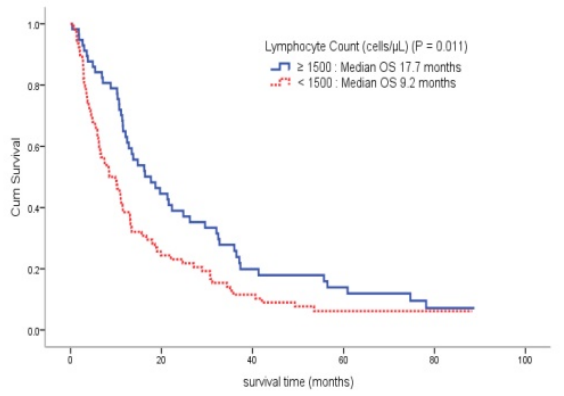

(E)

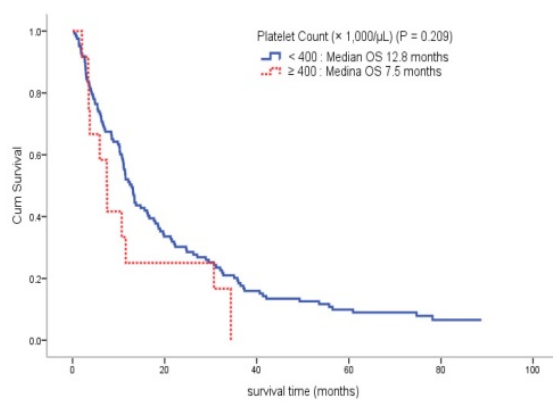

(C)

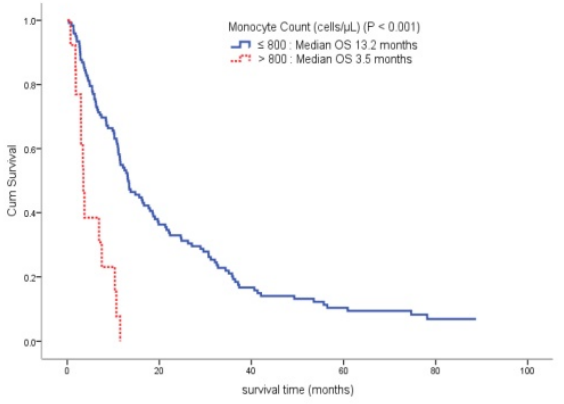

(F)

Figure 2. Overall survival curve of patients according to hemoglobin (A) and ferritin (B) concentrations, as well as platelet (C), neutrophil (D), lymphocyte (E), and monocyte $(F)$ counts.

Univariate analysis was performed to identify the predictable factors for multivariable analysis of proportional hazards regression. Among the clinicopathologic factors, age (hazard ratio [HR], 1.02; 95\% confidence interval [CI], 1.01-1.04; $p<0.001)$, ECOG-PS score (HR, 2.01; 95\% CI, 1.40-2.88; $p<0.001$ ) influenced the survival outcomes, whereas stage and cell type showed no impact on survival. Regarding host-related biomarkers, a lack of anemia was associated with prolonged survival (HR, 0.69; 95\% CI, $0.48-0.99 ; p=0.046)$. Patients with increased ANC showed significantly poorer survival outcome (HR, 
3.41; 95\% CI, 2.02-5.75; $p<0.001)$. High ALC was associated with decreased survival hazard (HR, 0.63; 95\% CI, 0.44-0.90; $p=0.011)$. Increased AMC was associated with an increased risk of mortality (HR, 4.40; 95\% CI, 2.37-8.16; $p<0.001)$. High ferritin levels were significantly associated with poor survival outcomes (HR, 1.91; 95\% CI, 1.33-2.73; $p<0.001)$. The presence of anemia, neutrophilia, lymphocytopenia, monocytosis, and hyperferritinemia was associated with significantly poor survival outcomes (Table 2).

Stepwise multivariate analysis was performed using these significant host-related biomarkers. In this analysis, ALC, AMC, and serum ferritin concentration were independently associated with survival. Increased AMC (HR, 3.15; 95\% CI, 1.64-6.04; $p<0.001)$ and increased serum ferritin concentration (HR, 1.81; 95\% CI, 1.24-2.64; $p=0.002$ ) were significantly associated with poor survival outcome, whereas increased ALC was associated with prolonged survival (HR, 0.57; 95\% CI, 0.40-0.83; $p=0.004$ ) (Table 2).
Pearson's correlation coefficient analysis of the independent prognostic biomarkers revealed that AMC and ALC were very weakly correlated $(r=0.17$, $p=0.048)$, AMC and ferritin were weakly positively correlated $(r=0.25, p=0.004)$, and ALC and ferritin were weakly negatively associated $(r=-0.22, p=$ 0.012). However, among biomarkers, ANC and AMC $(r=0.64, p<0.001)$ were strongly correlated, while ANC and ferritin $(r=0.45, p<0.001)$ were moderately correlated (Figure 3). The baseline clinicopathologic characteristics and the biomarker value grouped according to ferritin levels are shown in Table 3. The distribution of age, sex, cancer stage, cell type, and number of organs with metastasis was similar between the two groups. Patients with increased ferritin concentration were more likely to have a poorer ECOG-PS score $(p=0.002)$, more chemotherapy $(p=0.031)$ and radiotherapy $(p=0.024)$ regimens, elevated ANC $(p=0.002)$ and $\operatorname{AMC}(p=$ $0.013)$, lower levels of $\mathrm{Hb}(p=0.011)$, and decreased ALC $(p=0.017)$ (Table 3$)$.

Table 2. The prognostic impacts of host-related biomarkers by Cox-proportional hazard regression analysis.

\begin{tabular}{|c|c|c|c|c|c|c|c|}
\hline \multirow[t]{2}{*}{ Variable } & \multirow[t]{2}{*}{ Unit } & \multicolumn{3}{|c|}{ Univariate Analysis } & \multicolumn{3}{|c|}{ Multivariate Analysis } \\
\hline & & HR & 95\% HR CI & $P$-value & HR & 95\% HR CI & $P$-value \\
\hline \multicolumn{8}{|l|}{ Clinicopathologic factors } \\
\hline Age & 1 & 1.02 & $1.01-1.04$ & $<0.001$ & & & \\
\hline Sex & Male/female & 0.73 & $0.51-1.04$ & 0.083 & & & \\
\hline ECOG-PS & $0-1 / 2$ & 2.01 & $1.40-2.88$ & $<0.001$ & & & \\
\hline Stage & IIIB/IV & 1.12 & $0.59-2.14$ & 0.734 & & & \\
\hline Cell type & Non-squamous cell carcinoma/ squamous cell carcinoma & 1.44 & $0.93-2.23$ & 0.106 & & & \\
\hline \multicolumn{8}{|l|}{ Host-related biomarkers } \\
\hline \multirow[t]{2}{*}{ Hemoglobin $(\mathrm{g} / \mathrm{dL})$} & Men $<13 / \geq 13$ & 0.69 & $0.48-0.99$ & 0.046 & & & \\
\hline & Women $<12 / \geq 12$ & & & & & & \\
\hline \multirow[t]{2}{*}{ Ferritin (ng/mL) } & Men $\leq 200 />200$ & 1.91 & $1.33-2.73$ & $<0.001$ & 1.81 & $1.24-2.64$ & 0.002 \\
\hline & Women $\leq 150 />150$ & & & & & & \\
\hline Platelet $\left(\times 10^{3} / \mu \mathrm{L}\right)$ & $<400 / \geq 400$ & 1.49 & $0.80-2.78$ & 0.212 & & & \\
\hline Neutrophil (cells/ $\mu \mathrm{L})$ & $\leq 7700 />7700$ & 3.41 & $2.02-5.75$ & $<0.001$ & & & \\
\hline Lymphocyte (cells/ $\mu \mathrm{L}$ ) & $<1500 / \geq 1500$ & 0.63 & $0.44-0.90$ & 0.011 & 0.57 & $0.40-0.83$ & 0.004 \\
\hline Monocyte $($ cells $/ \mu \mathrm{L})$ & $\leq 800 />800$ & 4.40 & $2.37-8.16$ & $<0.001$ & 3.15 & $1.64-6.04$ & $<0.001$ \\
\hline
\end{tabular}

Table 3. Characteristics of patients according to ferritin concentration.

\begin{tabular}{|c|c|c|c|}
\hline & Ferritin $(N=67)$ & Ferritin $(N=68)$ & $P$-value \\
\hline & Men $\leq 200$, Women $\leq 150 \mathrm{ng} / \mathrm{mL}$ & Men $>200$, Women $>150 \mathrm{ng} / \mathrm{mL}$ & \\
\hline \multicolumn{4}{|l|}{ Clinicopathologic factors } \\
\hline Age & $63[31-87]^{*}$ & 59.5 [39-83] & 0.694 \\
\hline Sex (Male/Female) & $35 / 32(52.2 / 47.8) \dagger$ & $43 / 25(63.2 / 36.8)$ & 0.196 \\
\hline $\operatorname{ECOG}(0 / 1 / 2)$ & $5 / 44 / 18(7.5 / 65.7 / 26.9)$ & $3 / 26 / 39(4.4 / 38.2 / 57.4)$ & 0.002 \\
\hline Stage (IIIB/IV) & $8 / 59(11.9 / 88.1)$ & $4 / 64(5.9 / 94.1)$ & 0.216 \\
\hline Cell type (adenocarcinoma/squamous cell carcinoma/others) & $46 / 14 / 7(68.7 / 20.9 / 10.4)$ & $46 / 15 / 7(67.6 / 22.1 / 10.3)$ & 0.987 \\
\hline Number of organs with metastasis $(0 / 1 / 2 / \geq 3)$ & $8 / 32 / 17 / 10(11.9 / 47.8 / 25.4 / 14.9)$ & $4 / 22 / 22 / 20(5.9 / 32.4 / 32.4 / 29.4)$ & 0.067 \\
\hline Prior surgery & $49 / 18(73.1 / 26.9)$ & $53 / 15(77.9 / 22.1)$ & 0.516 \\
\hline Prior chemotherapy & $35 / 32(52.2 / 47.8)$ & $23 / 45(33.8 / 66.2)$ & 0.031 \\
\hline Prior radiation therapy & $49 / 18(73.1 / 26.9)$ & $37 / 31(54.4 / 45.6)$ & 0.024 \\
\hline \multicolumn{4}{|l|}{ Host-related biomarkers } \\
\hline Hemoglobin $(\mathrm{g} / \mathrm{dL})$ & $12.3[9.0-14.9]$ & $11.5[6.8-14.8]$ & 0.011 \\
\hline Platelet $\left(\times 10^{3} / \mu \mathrm{L}\right)$ & $243.0[104.0-591.0]$ & $259.0[77.0-540.0]$ & 0.271 \\
\hline Neutrophil (cells/ $\mu \mathrm{L}$ ) & 3763.2 [1075.8-10651.5] & 5199.1 [1598.0-19680.0] & 0.002 \\
\hline Lymphocyte (cells/ $\mu \mathrm{L})$ & $1600.0[427.0-3465.0]$ & $1189.8[78.6-3361.6]$ & 0.017 \\
\hline Monocyte (cells $/ \mu \mathrm{L})$ & 470.4 [159.0-963.2] & $541.3[42.0-1920.0]$ & 0.013 \\
\hline
\end{tabular}

*median [range], $\uparrow \mathrm{N}(\%)$ 


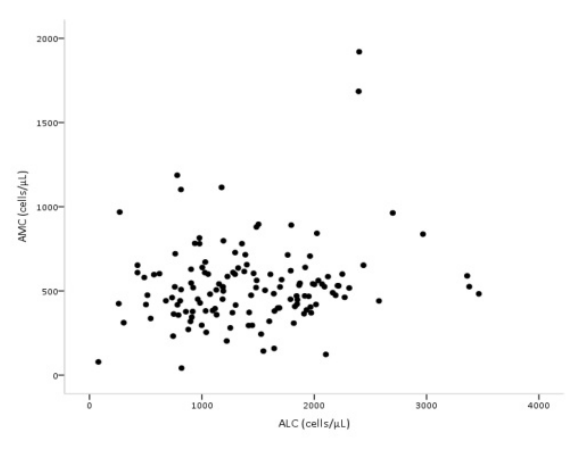

(A)

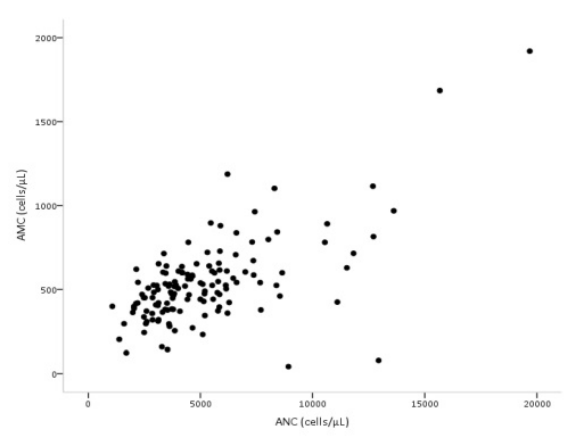

(D)

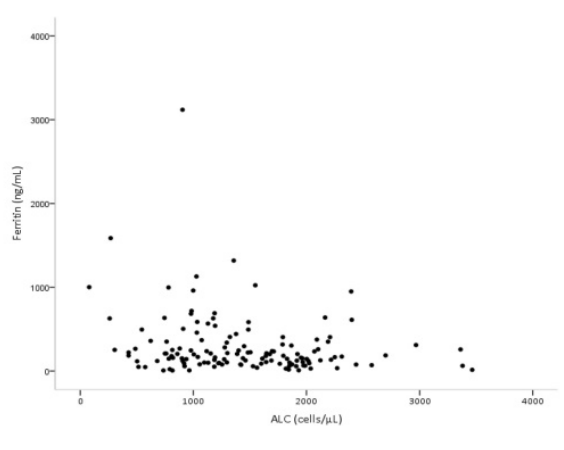

(B)

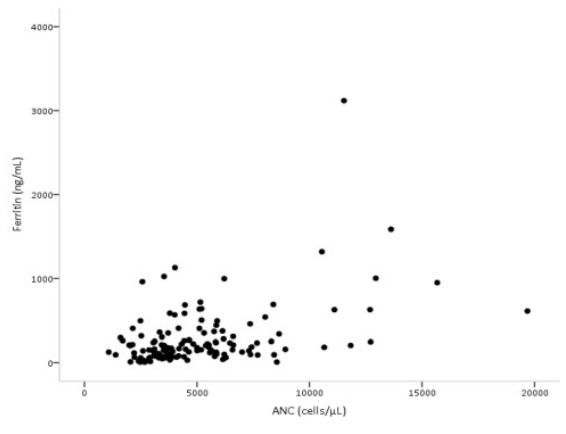

(E)

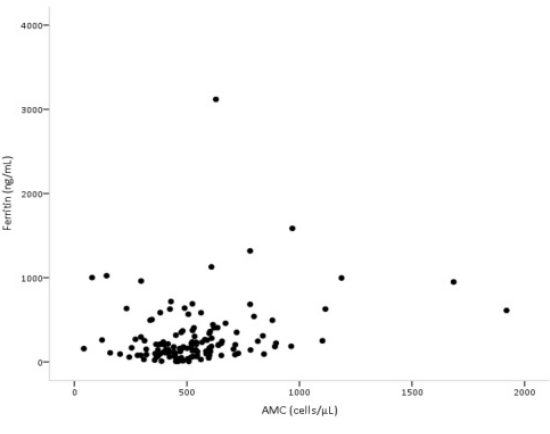

(C)

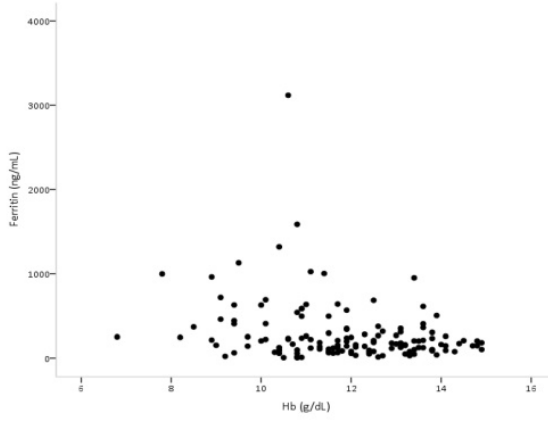

(F)

Figure 3. Scatter plots showing the correlations between (A) ALC and AMC, (B) ALC and ferritin, (C) AMC and ferritin, (D) ANC and AMC, (E) ANC and ferritin, and $(\mathrm{F}) \mathrm{Hb}$ and ferritin.

\section{Discussion}

For decades, platinum-based doublets with 10 months of life expectancy were recommended as the first-line therapy for patients with advanced NSCLC. ${ }^{26}$ Recently, immunotherapy using programmed death 1 antibodies has been recommended as the first-line regimen for patients with programmed death-ligand 1 expression levels over $50 \%$, based on 10.3 months of PFS in advanced NSCLC. 27 Due to the promising outcome of immunotherapy, the paradigm of cancer therapy is shifting toward host-based therapy. Despite this progress, advanced NSCLC with high mortality is still an intractable disease in clinical practice. The precise prediction of prognosis is an important issue when selecting the optimal treatment, including the continuation of systemic therapy, palliative care, and supportive care. Thus, it is critical to identify available biomarkers that predict treatment response and reflect survival time in advanced NSCLC.

To date, the anatomical extent of tumor and the response to therapy have been the commonly used prognostic factors in clinical practice and the majority of studies on the prognosis of NSCLC have focused on tumor or treatment-related factors. ${ }^{12,28}$ Most biomarker studies have been performed in early-stage patients or at the initiation of treatment, immediately after diagnosis. Predicting prognosis, especially in advanced NSCLC, should be approached differently from early-stage NSCLC.

One of the basic biomarkers is blood cell count, which indicates the general condition of patients with solid tumors as well as with hematologic malignancies. ${ }^{29}$ Hematologic changes in malignant disease, especially in the advanced stages, may be associated with a poor prognosis. Changes in total white blood cell count, hemoglobin level, and PLT count are frequently observed in the progression of cancer or as an effect of systemic therapy. Hematologic abnormalities such as pancytopenia, anemia, a variety of white blood cell including leucopenia or leukocytosis, and platelet and blood coagulation abnormalities are associated with systemic inflammation or widespread tumor progression. Among these hematologic reactions in advanced cancer, this study focused on the prognostic impact of anemia, thrombocytosis, neutrophilia, lymphocytopenia, monocytosis, and hyperferritinemia as host-related prognostic factors in advanced NSCLC.

The prognostic effect of thrombocytosis on 
survival in lung cancer is controversial due to studies reporting conflicting findings. ${ }^{14,30,31}$ In the current study, the presence of thrombocytosis was not associated with a significant difference in survival time; thus, thrombocytosis was not a significant prognostic factor for survival. This result indicated that thrombocyte was the least associated parameter to survival among blood cell assessments in advanced NSCLC.

Anemia is one of the most frequently observed hematologic manifestations in both solid tumors and hematologic malignancies. Although high $\mathrm{Hb}$ levels have been reported to be a significant favorable factor for survival in extensive stage NSCLC, ${ }^{32}$ there have been conflicting reports on the relationship between $\mathrm{Hb}$ level and survival time in NSCLC patients. ${ }^{33-36}$ In the current study, the presence of anemia was significantly associated with short survival and increased risk of mortality, but anemia was not a significant independent prognostic factor.

Studies on leukocytes as a prognostic factor in NSCLC mostly focus on total white blood cell counts, leukocyte ratios such as neutrophil-lymphocyte ratios, or comparisons of white blood cell count with inflammation biomarkers. Only one previous study on the association between these leukocyte types and advanced NSCLC reported elevated ANC as an independent prognostic factor. ${ }^{37}$ However, the current study evaluated leukocyte subtypes as representatives of the immune system, such as ANC, ALC, and AMC in the peripheral blood in order to identify basic changes associated with hematologic abnormalities. Based on the results, increased ANC was significantly associated with short survival and increased risk of mortality, but the impact of ANC as an independent prognostic factor for survival was not statistically significant. The reduced ALC was associated with poor survival and increased risk of mortality, while lymphocytopenia was an independent prognostic factor for survival in advanced NSCLC. Increased AMC was strongly associated with poor survival and increased risk of mortality in this study. In contrast to neutrophilia, monocytosis was identified as a significant independent prognostic factor in advanced NSCLC. The hematologic changes associated with neutrophilia, lymphocytopenia, and monocytosis, which may be caused by the tumor or therapy, were significantly associated with decreased survival time and increased risk of mortality in advanced NSCLC.

Recently, a detailed interaction between the immune system and cancer was proposed, which included seven parameters: general immune status, immune cell infiltration, absence of checkpoints, absence of soluble inhibitors, absence of inhibitory tumor metabolism, tumor sensitivity to immune effectors, and tumor foreignness. ${ }^{38}$ Among these parameters, the basic biomarkers that reflect the general patient immune status include the leukocytes subgroups of neutrophils, lymphocytes, and monocytes, which were assessed in this study. Regarding the immune cell infiltration, the role of tumor infiltration lymphocytes (TILs), tumor-associated macrophages (TAMs), and neutrophil extracellular traps (NETs) reportedly influence cancer progression and patient survival. High levels of TILs were reported to decrease the risk of lung cancer recurrence, ${ }^{39}$ while TAMs promote cancer cell growth, invasion, metastasis, and immunosuppression. ${ }^{40}$ Recently, NETs were identified as sequester circulating tumor cells that promoted metastasis. ${ }^{41}$ Leukocytes originate to regulate tissue homeostasis and play a role in wound healing, but an abundance of innate and adaptive immune cells can lead to chronic inflammation, which can enhance cancer development. Thus, leukocytes may act paradoxically as mediators of cancer progression. ${ }^{42}$

Ferritin stores iron, therefore, ferritin levels have been used to indicate the total amount of iron in the body. In chronic diseases, high serum ferritin concentrations are often indicative of anemia. Ferritin as an acute phase response protein has been associated with the pathological processes of inflammation and infection..$^{43}$ Recent studies have also identified that increased ferritin concentrations indicate the pathological processes of proliferation, angiogenesis, and immunosuppression. ${ }^{44,45}$ Regarding the subunits, serum ferritin is predominantly composed of L-ferritin under normal physiological conditions, whereas high $\mathrm{H}$-ferritin serum levels, or a high ratio of $\mathrm{H}$ - to L-ferritins, are present under malignant conditions. ${ }^{46}$ Although the mechanism of this phenomenon remains unclear, studies have demonstrated that $\mathrm{H}$-ferritin plays a role in malignancy and could be a potential biomarker for the detection of cancer. ${ }^{46}$ Since the relationship between ferritin and cancer was first reported, ${ }^{47}$ elevated serum ferritin levels have been associated with breast cancer, hepatocellular carcinoma, pancreatic cancer, colorectal cancer, and hematologic malignancies. ${ }^{48-52}$

Increased ferritin level combined with carcinoembryonic antigens could be a prognostic factor in elderly lung cancer patients. ${ }^{53}$ Based on predicting prognosis, ferritin was not a useful marker for staging and monitoring, ${ }^{54}$ but it might have prognostic potential in small cell lung cancer and primary lung cancer. ${ }^{55,56}$ One study reported a higher response rate to platinum-based chemotherapy in the 
group with normal ferritin levels than in the group with higher ferritin levels. ${ }^{57}$ Until now, no previous study had assessed the prognostic impact of serum ferritin for OS in advanced NSCLC. Based on the results of current study, high ferritin level was significantly associated with reduced survival time and increased risk of mortality. The prognostic impact of ferritin for survival was more significant than other known prognostic factors such as ANC, $\mathrm{Hb}$ level, or platelet count. As an independent prognostic factor for survival, hyperferritinemia was significant enough a predictor to compete with lymphocytopenia and monocytosis.

The correlations among the independent prognostic biomarkers for survival revealed weak associations, while ANC and AMC levels were strongly correlated and ANC and ferritin levels were moderately correlated. The correlation between ANC and $\mathrm{AMC}$ reveals the pathophysiological reaction of leukocytes and the association between ANC and ferritin may reflect pathologic changes in chronic inflammation or cancer progression. However, in the multivariate analysis modeling, ANC, the main factor of correlation between biomarkers, was removed because of its weak impact on survival. To examine the impact of ferritin, the patients were grouped according to ferritin levels and the biomarker values were compared between these groups. Patients with an increased ferritin level were more likely to have increased $\mathrm{ANC}$ and $\mathrm{AMC}$ values and decreased $\mathrm{Hb}$ and ALC values.

In this study, we confirmed the prognostic efficacy of ALC and AMC and identified serum ferritin as a significant prognostic factor for survival. The level of serum ferritin was a strong survival predictor comparable to leukocyte ALC and AMC, which represent the host immune system.

This finding implies that the parameters that present various kinds of host-related information were identified as crucial prognostic factors of tumor progression as well as survival. Furthermore, the relationship between ferritin and leukocytes seems to be relevant to the pathophysiology of cancer, such as inflammation, immunity, oxidative stress, and tumor progression as a manifestation of advanced NSCLC. To interpret the mechanism underlying these findings, biological studies of serum ferritin and immune cells with various approaches are necessary, and changes in immune-related biological markers according to tumor progression should be assessed in translational research. Particularly, novel functions of $\mathrm{H}$-ferritin, which relate to mechanisms of carcinogenesis and regulators of the immune system, should be studied in biomedical translational research to find correlations between laboratory medicine and clinical practice. In addition, well-designed prospective clinical trials are needed to confirm these findings and to support translational studies.

Owing to the retrospective review of medical records, this study was limited by the small number of patients who underwent ferritin testing, the lack of a comparison group, potential confounding factors of the data, and the limited number of prognostic factors available for analysis.

Despite these limitations, this study identified lymphocyte and monocyte counts as well as ferritin concentration as host-related independent prognostic factors that significantly affected survival in patients with advanced NSCLC.

\section{Acknowledgements}

The authors are grateful to the clinic patients and clinicians.

\section{Institutional review board statement}

This study was reviewed and approved by the institutional review board of Kyung Hee University Hospital at Gangdong (IRB no: KHNMCOH 2016-02001).

\section{Informed consent statement}

The need for informed consent was waived because the analysis used anonymous clinical data that were obtained.

\section{Competing Interests}

The authors have declared that no competing interest exists.

\section{References}

1. Torre LA, Bray F, Siegel RL, Ferlay J, Lortet-Tieulent J, Jemal A. Global cancer statistics, 2012. CA Cancer J Clin. 2015.

2. Sher T, Dy GK, Adjei AA. Small cell lung cancer. Mayo Clinic proceedings. 2008;83(3):355-367.

3. Spiro SG, Gould MK, Colice GL, American College of Chest P. Initial evaluation of the patient with lung cancer: symptoms, signs, laboratory tests, and paraneoplastic syndromes: ACCP evidenced-based clinical practice guidelines (2nd edition). Chest. 2007;132(3 Suppl):149S-160S

4. Janku F, Stewart DJ, Kurzrock R. Targeted therapy in non-small-cell lung cancer--is it becoming a reality? Nature reviews Clinical oncology. 2010;7(7):401-414.

5. SEER N. Cancer Stat Facts: Lung and Bronchus Cancer. 2016; https://seer.cancer.gov/statfacts/html/lungb.html.

6. Melosky B, Chu Q, Juergens R, Leighl N, McLeod D, Hirsh V. Pointed Progress in Second-Line Advanced Non-Small-Cell Lung Cancer: The Rapidly Evolving Field of Checkpoint Inhibition. J Clin Oncol. 2016;34(14):1676-1688.

7. Pardoll DM. The blockade of immune checkpoints in cancer immunotherapy. Nat Rev Cancer. 2012;12(4):252-264.

8. Sukari A, Nagasaka M, Al-Hadidi A, Lum LG. Cancer Immunology and Immunotherapy. Anticancer Res. 2016;36(11):5593-5606.

9. Swanton C, Govindan R. Clinical Implications of Genomic Discoveries in Lung Cancer. The New England journal of medicine. 2016;374(19):1864-1873.

10. Booth CM, Eisenhauer EA. Progression-free survival: meaningful or simply measurable? J Clin Oncol. 2012;30(10):1030-1033.

11. Goodwin PJ, Meyerhardt JA, Hursting SD. Host factors and cancer outcome. J Clin Oncol. 2010;28(26):4019-4021.

12. Brundage MD, Davies D, Mackillop WJ. Prognostic factors in non-small cell lung cancer: a decade of progress. Chest. 2002;122(3):1037-1057.

13. WHO. Hemoglobin concentrations for the diagnosis of anaemia and assessment of severity. 2011; http://apps.who.int/iris/bitstream/10665/ 85839/3/WHO_NMH_NHD_MNM_11.1_eng.pdf?ua=1. 
14. Maraz A, Furak J, Varga Z, Kahan Z, Tiszlavicz L, Hideghety K. Thrombocytosis has a negative prognostic value in lung cancer. Anticancer Res. 2013;33(4):1725-1729.

15. Ozcan C, Telli O, Ozturk E, et al. The prognostic significance of preoperative leukocytosis and neutrophil-to-lymphocyte ratio in patients who underwent radical cystectomy for bladder cancer. Can Urol Assoc J. 2015;9(11-12):E789-794.

16. Carli L, Tani C, Vagnani S, Signorini V, Mosca M. Leukopenia, lymphopenia, and neutropenia in systemic lupus erythematosus: Prevalence and clinical impact--A systematic literature review. Semin Arthritis Rheum. 2015;45(2):190-194.

17. Bari A, Tadmor $\mathrm{T}$, Sacchi $\mathrm{S}$, et al. Monocytosis has adverse prognostic significance and impacts survival in patients with T-cell lymphomas. Leuk Res. 2013;37(6):619-623

18. WHO. Serum ferritin concentrations for the assessment of iron status and iron deficiency in populations. 2011; http://www.who.int/vmnis/indicators/ serum_ferritin.pdf.

19. Choi WC LJ, Lee EO, et al. . Study on antiangiogenic and antitumor activities of processed Rhus Verniciflua Stokes extract. Korean J Oriental Physiology $\mathcal{E}$ Pathology. 2006;20:1-5.

20. Kim JH, Kim HP, Jung $\mathrm{CH}$, et al. Inhibition of cell cycle progression via p27Kip1 upregulation and apoptosis induction by an ethanol extract of Rhus verniciflua Stokes in AGS gastric cancer cells. Int J Mol Med. 2006;18(1):201-208.

21. Lee SK, Jung HS, Eo WK, Lee SY, Kim SH, Shim BS. Rhus verniciflua Stokes extract as a potential option for treatment of metastatic renal cell carcinoma: report of two cases. Ann Oncol. 2010;21(6):1383-1385.

22. Lee JH, Lee HJ, Lee HJ, et al. Rhus verniciflua Stokes prevents cisplatin-induced cytotoxicity and reactive oxygen species production in MDCK-I renal cells and intact mice. Phytomedicine : international journal of phytotherapy and phytopharmacology. 2009;16(2-3):188-197.

23. Park JH, Moon G. Effect of allergen removed Rhus verniciflua extract on inhibition of tumor metastasis. J of Kor Traditional Oncology. 2010;15(1):47-61.

24. Lee J, Chae J, Lee S, et al. The efficacy and safety of standardized allergen-removed Rhus verniciflua extract as maintenance therapy after first-line chemotherapy in patients with advanced non-small cell lung cancer. The American journal of Chinese medicine. 2013:41(4):773-787.

25. Cheon SH, Kim KS, Kim S, Jung HS, Choi WC, Eo WK. Efficacy and safety of Rhus verniciflua stokes extracts in patients with previously treated advanced non-small cell lung cancer. Forschende Komplementarmedizin. 2011;18(2):77-83.

26. Schiller $\mathrm{JH}$, Harrington $\mathrm{D}$, Belani $\mathrm{CP}$, et al. Comparison of four chemotherapy regimens for advanced non-small-cell lung cancer. The New England journal of medicine. 2002;346(2):92-98

27. Reck M, Rodriguez-Abreu D, Robinson AG, et al. Pembrolizumab versus Chemotherapy for PD-L1-Positive Non-Small-Cell Lung Cancer. The New England journal of medicine. 2016;375(19):1823-1833.

28. Liang W, He J, Shen Y, et al. Impact of Examined Lymph Node Count on Precise Staging and Long-Term Survival of Resected Non-Small-Cell Lung Cancer: A Population Study of the US SEER Database and a Chinese Multi-Institutional Registry. I Clin Oncol. 2017;35(11):1162-1170.

29. Shahid S. Review of hematological indices of cancer patients receiving combined chemotherapy \& radiotherapy or receiving radiotherapy alone. Crit Rev Oncol Hematol. 2016;105:145-155.

30. Pedersen LM, Milman N. Prognostic significance of thrombocytosis in patients with primary lung cancer. Eur Respir J. 1996;9(9):1826-1830.

31. Cakar B, Karaoglanoglu M, Sayici Y, Gonullu Demirag G, Yucel I. The prognostic value of thrombocytosis in newly diagnosed lung cancer patients: a retrospective analysis. J BUON. 2011;16(4):677-681.

32. Albain KS, Crowley JJ, LeBlanc M, Livingston RB. Survival determinants in extensive-stage non-small-cell lung cancer: the Southwest Oncology Group experience. J Clin Oncol. 1991;9(9):1618-1626.

33. Tomita M, Shimizu T, Hara M, Ayabe T, Onitsuka T. Impact of preoperative hemoglobin level on survival of non-small cell lung cancer patients. Anticancer Res. 2008;28(3B):1947-1950.

34. Jazieh AR, Hussain M, Howington JA, et al. Prognostic factors in patients with surgically resected stages I and II non-small cell lung cancer. Ann Thorac Surg. 2000;70(4):1168-1171

35. Gauthier I, Ding K, Winton T, et al. Impact of hemoglobin levels on outcomes of adjuvant chemotherapy in resected non-small cell lung cancer: the JBR.10 trial experience. Lung cancer. 2007;55(3):357-363.

36. MacRae R, Shyr Y, Johnson D, Choy H. Declining hemoglobin during chemoradiotherapy for locally advanced non-small cell lung cancer is significant. Radiotherapy and oncology : journal of the European Society for Therapeutic Radiology and Oncology. 2002;64(1):37-40.

37. Teramukai S, Kitano T, Kishida Y, et al. Pretreatment neutrophil count as an independent prognostic factor in advanced non-small-cell lung cancer: an analysis of Japan Multinational Trial Organisation LC00-03. Eur J Cancer. 2009:45(11):1950-1958

38. Blank CU, Haanen JB, Ribas A, Schumacher TN. CANCER IMMUNOLOGY. The "cancer immunogram". Science. 2016;352(6286):658-660.

39. Kilic A, Landreneau RJ, Luketich JD, Pennathur A, Schuchert MJ. Density of tumor-infiltrating lymphocytes correlates with disease recurrence and survival in patients with large non-small-cell lung cancer tumors. J Surg Res. 2011;167(2):207-210.

40. Lahmar Q, Keirsse I, Laoui D, Movahedi K, Van Overmeire E, Van Ginderachter JA. Tissue-resident versus monocyte-derived macrophages in the tumor microenvironment. Biochim Biophys Acta. 2016;1865(1):23-34.
41. Cools-Lartigue J, Spicer J, McDonald B, et al. Neutrophil extracellular traps sequester circulating tumor cells and promote metastasis. J Clin Invest. 2013.

42. de Visser KE, Eichten A, Coussens LM. Paradoxical roles of the immune system during cancer development. Nat Rev Cancer. 2006;6(1):24-37.

43. Cazzola M, Arosio P, Bellotti V, et al. Immunological reactivity of serum ferritin in patients with malignancy. Tumori. 1985;71(6):547-554.

44. Coffman LG, Parsonage D, D'Agostino R, Jr., Torti FM, Torti SV. Regulatory effects of ferritin on angiogenesis. Proc Natl Acad Sci U S A. 2009;106(2):570-575.

45. Alkhateeb AA, Han B, Connor JR. Ferritin stimulates breast cancer cells through an iron-independent mechanism and is localized within tumor-associated macrophages. Breast Cancer Res Treat. 2013;137(3):733-744.

46. Fan K, Gao L, Yan X. Human ferritin for tumor detection and therapy. Wiley Interdiscip Rev Nanomed Nanobiotechnol. 2013;5(4):287-298.

47. Hazard JT, Drysdale JW. Ferritinaemia in cancer. Nature. 1977;265(5596):755-756.

48. Jezequel P, Campion L, Spyratos F, et al. Validation of tumor-associated macrophage ferritin light chain as a prognostic biomarker in node-negative breast cancer tumors: A multicentric 2004 national PHRC study. Int I Cancer. 2012;131(2):426-437.

49. Kalousova M, Krechler T, Jachymova M, Kubena AA, Zak A, Zima T. Ferritin as an independent mortality predictor in patients with pancreas cancer. Results of a pilot study. Tumour Biol. 2012;33(5):1695-1700.

50. Facciorusso A, Del Prete V, Antonino M, et al. Serum ferritin as a new prognostic factor in hepatocellular carcinoma patients treated with radiofrequency ablation. J Gastroenterol Hepatol. 2014;29(11):1905-1910.

51. Zhang XZ, Su AL, Hu MQ, Zhang XQ, Xu YL. Elevated serum ferritin levels in patients with hematologic malignancies. Asian Pac I Cancer Prev. 2014;15(15):6099-6101.

52. Lee S, Song A, Eo W. Serum Ferritin as a Prognostic Biomarker for Survival in Relapsed or Refractory Metastatic Colorectal Cancer. I Cancer. 2016;7(8):957-964.

53. Ji M, Li XD, Shi HB, et al. Clinical significance of serum ferritin in elderly patients with primary lung carcinoma. Tumour Biol. 2014;35(10):10195-10199.

54. Ferrigno D, Buccheri G. Serum ferritin levels in lung cancer patients. Eur I Cancer. 1992;28(1):241.

55. Milman N, Sengelov H, Dombernowsky P. Iron status markers in patients with small cell carcinoma of the lung. Relation to survival. $\mathrm{Br} J$ Cancer. 1991;64(5):895-898.

56. Milman N, Pedersen LM. The serum ferritin concentration is a significant prognostic indicator of survival in primary lung cancer. Oncol Rep. 2002;9(1):193-198.

57. Shi HB, Li XD, Jiang JT, Zhao WQ, Ji M, Wu CP. Serum ferritin is elevated in advanced non-small cell lung cancer patients and is associated with efficacy of platinum-based chemotherapy. J Cancer Res Ther. 2014;10(3):681-685. 